\title{
REMARKS FOR BASIC APPELL SERIES
}

\author{
GYEONG-SIG SEO AND JOONG-SOO PARK
}

\begin{abstract}
Let $k$ be an imaginary quadratic field, $\mathfrak{H}$ the complex upper half plane, and let $\tau \in k \cap \mathfrak{H}, q=\exp (\pi i \tau)$. And let $n, t$ be positive integers with $1 \leq t \leq n-1$. Then $q^{\frac{n}{12}-\frac{t}{2}+\frac{t^{2}}{2 n}} \prod_{m=1}^{\infty}\left(1-q^{n m-t}\right)\left(1-q^{n m-(n-t)}\right)$ is an algebraic number [10]. As a generalization of this result, we find several infinite series and products giving algebraic numbers using Ramanujan's ${ }_{1} \psi_{1}$ summation. These are also related to Rogers-Ramanujan continued fractions.
\end{abstract}

\section{Introduction}

Ramanujan developed $q$-series and theta functions and discovered several new and profound theroems in the theory of theta functions. This paper is related to the Ramanujan theta function

(1.1) $f(a, b)=1+\sum_{m=1}^{\infty}(a b)^{\frac{m(m-1)}{2}}\left(a^{m}+b^{m}\right)=\sum_{m=-\infty}^{\infty} a^{\frac{m(m+1)}{2}} b^{\frac{m(m-1)}{2}}$,

where $|a b|<1$. To facilitate the product representations of the theta functions, we introduce the standard notations;

$$
\begin{array}{ll}
(a)_{0} & :=(a ; q)_{0}:=1, \\
(a)_{n} & :=(a ; q)_{n}:=(1-a)(1-a q) \cdots\left(1-a q^{n-1}\right), n \geq 1, \\
(a)_{\infty} & :=(a ; q)_{\infty}:=\lim _{n \rightarrow \infty}(a ; q)_{n},|q|<1 .
\end{array}
$$

Then Ramanujan theta function has an infinite product form:

$$
f(a, b)=(-a ; a b)_{\infty}(-b ; a b)_{\infty}(a b ; a b)_{\infty} .
$$

In this paper for an imaginary quadratic field $k$, we fix $\tau \in k \cap \mathfrak{H}$. Then $|q|<1$.

Received September 28, 2009. Accepted November 6, 2009.

2000 Mathematics Subject Classification: [2000]11Jxx, 11R04, 11F11.

Key words and phrases: Ramanujan theta function, quadratic field, algebraic number, Rogers-Ramanujan continued fraction, Appell series. 
For $\left|\frac{b}{a}\right|<|z|<1$, the following is called Ramanujan's ${ }_{1} \psi_{1}$ summation [2]:

$$
\sum_{n=-\infty}^{\infty} \frac{(a)_{n}}{(b)_{n}} z^{n}=\frac{(a z)_{\infty}(q / a z)_{\infty}(q)_{\infty}(b / a)_{\infty}}{(z)_{\infty}(b / a z)_{\infty}(b)_{\infty}(q / a)_{\infty}}
$$

Theorem 1.1. Let $k$ be an imaginary quadratic field and $\tau \in k \cap \mathfrak{H}$. For rational numbers $l$ and $t$, if $0<l-t<1$, then

$$
\begin{aligned}
& -q^{\frac{(t+l)(t-l+1)}{2}} \frac{\left(1-q^{l-t}\right)\left(1-q^{1-(l-t)}\right)}{\left(1-q^{l}\right)\left(1-q^{1-l}\right)\left(1-q^{t}\right)} \sum_{n, m=-\infty}^{\infty} \frac{\left(q^{t}\right)_{n}\left(q^{-t}\right)_{m}}{\left(q^{1+l}\right)_{n}\left(q^{2-l}\right)_{m}} q^{n+m} \\
= & q^{\frac{(t-l+2)(t+l-1)}{2}} \frac{1-q^{1-t} \sum_{n=-\infty}^{\infty} \frac{\left(q^{t}\right)_{n}}{\left(q^{l+1}\right)_{n}} q^{n}}{1-q^{l}} \frac{\left(q_{n=-\infty}^{\infty} \frac{\left(q^{1-l}\right)_{n}}{\left(q^{2-t}\right)_{n}}\right.}{\sum^{n}} \\
= & q^{\frac{t(t-1)-l(l-1)}{2}} \prod_{n=1}^{\infty} \frac{\left(1-q^{n-t}\right)\left(1-q^{n-1+t}\right)}{\left(1-q^{n-l}\right)\left(1-q^{n-1+l}\right)}
\end{aligned}
$$

is an algebraic number. In fact, it can be expressed as the product of Ramanujan theta functions:

$$
q^{\frac{t(t-1)-l(l-1)}{2}} \frac{f\left(-q^{t} ;-q^{1-t}\right)}{f\left(-q^{l} ;-q^{1-l}\right)} .
$$

Theorem 1.2. Let $k$ be an imaginary quadratic field and $\tau \in k \cap \mathfrak{H}$. For rational numbers $l$ and $t$, if $0<l, t, l+t<1$, then

$$
\begin{aligned}
& q^{\frac{t}{2}(2 l+t-1)} \sum_{n, m=0}^{\infty} \frac{\left(q^{t} ; q\right)_{n}\left(q^{-t} ; q\right)_{m}}{(q ; q)_{n}(q ; q)_{m}} q^{l(n-m)+m} \\
= & q^{\frac{t}{2}(2 l+t-1)} \frac{\sum_{n=0}^{\infty} \frac{\left(q^{t} ; q\right)_{n}}{(q ; q)_{n}} q^{l n}}{\sum_{n=0}^{\infty} \frac{\left(q^{t} ; q\right)_{n}}{(q ; q)_{n}} q^{(1-t-l) n}} \\
= & q^{\frac{t}{2}(2 l+t-1)} \prod_{n=1}^{\infty} \frac{\left(1-q^{n-t-l}\right)\left(1-q^{n-1+t+l}\right)}{\left(1-q^{n-l}\right)\left(1-q^{n-1+l}\right)}
\end{aligned}
$$

is an algebraic number. 
Theorem 1.3. Let $k$ be an imaginary quadratic field and $\tau \in k \cap \mathfrak{H}$. For rational numbers $l$ and $t$, if $0<l<1$, then

$$
\begin{gathered}
q^{\frac{1}{2} l(l+2 t-1)} \frac{\left(1-q^{l}\right)\left(1-q^{1-l}\right)}{\left(1-q^{t}\right)\left(1-q^{1-l-t}\right)\left(1-q^{l+t}\right)} \\
\sum_{m, n=-\infty}^{\infty} \frac{\left(q^{t}\right)_{m}\left(q^{-t}\right)_{n}}{\left(q^{1+t+l}\right)_{m}\left(q^{2-t-l}\right)_{n}} q^{l(n-m)+m} \\
=\quad q^{\frac{1}{2} l(l+2 t-1)} \frac{\left(1-q^{1-t}\right) \sum_{n=-\infty}^{\infty} \frac{\left(q^{t}\right)_{n}}{\left(q^{1+t+l}\right)_{n}} q^{l n}}{\left(1-q^{l+t}\right) \sum_{n=-\infty}^{\infty} \frac{\left(q^{1-t-l}\right)_{n}}{\left(q^{2-t}\right)_{n}} q^{l n}} \\
=q^{\frac{1}{2} l(l+2 t-1)} \prod_{n=1}^{\infty} \frac{\left(1-q^{n-t-l}\right)\left(1-q^{n-1+t+l}\right)}{\left(1-q^{n-t}\right)\left(1-q^{n-1+t}\right)}
\end{gathered}
$$

is an algebraic integer.

Using these identities of Theorem 1.1, 1.2 and 1.3, we present the relations between them and Rogers-Ramanujan continued fraction $R(\tau)$ :

$$
R(q)=\frac{q^{\frac{1}{5}}}{1+\frac{q}{1+\frac{q^{2}}{1+\cdots}}}, \quad|q|<1 .
$$

In [9], [10], D. Kim and J. K. Koo discussed and proved the expanded basic Appell series, and F. H. Jackson defined the four functions ([7], [8])

$$
\begin{aligned}
\Phi^{(1)}\left[a ; b, b^{\prime} ; c ; x, y ; q\right] & =\sum_{m=0}^{\infty} \sum_{n=0}^{\infty} \frac{(a)_{m+n}(b)_{m}\left(b^{\prime}\right)_{n}}{(c)_{m+n}(q)_{m}(q)_{n}} x^{m} y^{n} ; \\
\Phi^{(2)}\left[a ; b, b^{\prime} ; c, c^{\prime} ; x, y ; q\right] & =\sum_{m=0}^{\infty} \sum_{n=0}^{\infty} \frac{(a)_{m+n}(b)_{m}\left(b^{\prime}\right)_{n}}{(c)_{m}\left(c^{\prime}\right)_{n}(q)_{m}(q)_{n}} x^{m} y^{n} ; \\
\Phi^{(3)}\left[a, a^{\prime} ; b, b^{\prime} ; c ; x, y ; q\right] & =\sum_{m=0}^{\infty} \sum_{n=0}^{\infty} \frac{(a)_{m}\left(a^{\prime}\right)_{n}(b)_{m}\left(b^{\prime}\right)_{n}}{(c)_{m+n}(q)_{m}(q)_{n}} x^{m} y^{n} ; \\
\Phi^{(4)}\left[a ; b ; c, c^{\prime} ; x, y ; q\right] & =\sum_{m=0}^{\infty} \sum_{n=0}^{\infty} \frac{(a)_{m+n}(b)_{m+n}}{(c)_{m}\left(c^{\prime}\right)_{n}(q)_{m}(q)_{n}} x^{m} y^{n} .
\end{aligned}
$$

Also we can see the following identity due to G. E. Andrews [1]

$$
\Phi^{(1)}\left[b^{\prime} / x ; b, b^{\prime} ; b b^{\prime} ; x, y ; q\right]=\frac{(b x)_{\infty}\left(b^{\prime}\right)_{\infty}\left(b^{\prime} y / x\right)_{\infty}}{\left(b b^{\prime}\right)_{\infty}(x)_{\infty}(y)_{\infty}} .
$$

This will be a main tool for our result in $\S 5$. 


\section{Infinite products}

Let $\alpha=\left(\begin{array}{ll}a & b \\ 0 & d\end{array}\right)$ be a triangular matrix with integral entries and have determinant $|\alpha|$. And define

$$
\phi_{\alpha}(\tau):=|\alpha|^{12} \frac{\Delta\left(\alpha\left(\begin{array}{l}
\tau \\
1
\end{array}\right)\right)}{\Delta\left(\begin{array}{l}
\tau \\
1
\end{array}\right)}=|\alpha|^{12} d^{-12} \frac{\Delta(\alpha \tau)}{\Delta(\tau)}
$$

with $\Delta(\tau)=(2 \pi)^{12} q^{2} \prod_{m=1}^{\infty}\left(1-q^{2 m}\right)^{24}$. Then we recall the following facts.

Proposition 2.1. For any $\tau \in k \cap \mathfrak{H}$, the values $\phi_{\alpha}(\tau)$ are algebraic integers, which divide $|\alpha|^{12}$.

Proof. See the Theorem 2 and 4 of Chapter 12 in[12].

Proposition 2.2. Let $\tau \in k \cap \mathfrak{H}$. Then

$$
\begin{array}{cc}
\sqrt{2} q^{\frac{1}{24}} \prod_{m=1}^{\infty}\left(1+q^{m}\right), & q^{-\frac{1}{24}} \prod_{m=1}^{\infty}\left(1-q^{2 m-1}\right), \\
q^{-\frac{1}{24}} \prod_{m=1}^{\infty}\left(1+q^{2 m-1}\right) & \text { and } q^{-\frac{1}{24}} \prod_{m=1}^{\infty}\left(1+q^{m}\right)^{-1}
\end{array}
$$

are algebraic integers.

Proof. See Theorem 2.2 in [9].

Proposition 2.3. Let $k$ be an imaginary quadratic field. And let $n$ be a positive integer and $a$ be an integer such that $1 \leq a \leq n-1$. If $q^{t_{a}} \prod_{m=1}^{\infty}(1-$ $\left.q^{n m-a}\right)\left(1-q^{n m-(n-a)}\right)$ is a nonzero algebraic number for each $\tau \in k \cap \mathfrak{H}$, then

$$
q^{t_{a}} \prod_{m=1}^{\infty}\left(1+q^{n m-a}\right)\left(1+q^{n m-(n-a)}\right)
$$

is also an algebraic number.

Proof. Since $2 \tau \in k \cap \mathfrak{H}, q^{2 t_{a}} \prod_{m=1}^{\infty}\left(1-q^{2 n m-2 a}\right)\left(1-q^{2 n m-2(n-a)}\right)$ is an algebraic integer.

Thus the following

$q^{t_{a}} \prod_{m=1}^{\infty}\left(1+q^{n m-a}\right)\left(1+q^{n m-(n-a)}\right)=\frac{q^{2 t_{a}} \prod_{m=1}^{\infty}\left(1-q^{2 n m-2 a}\right)\left(1-q^{2 n m-2(n-a)}\right)}{q^{t_{a}} \prod_{m=1}^{\infty}\left(1-q^{n m-a}\right)\left(1-q^{n m-(n-a)}\right)}$

is an algebraic number. 


\section{Klein forms}

For an integer $N>6$ and an integer $r$ which is not a multiple of $N$, let $X_{r}(\tau)$ be the function defined by

$$
X_{r}(\tau)=X_{r}(\tau, N)=e^{-2 \pi i \frac{(r-1)(N-1)}{4 N}} \prod_{s=0}^{N-1} \frac{K_{r, s}(\tau)}{K_{1, s}(\tau)},
$$

where $K_{u, v}(\tau)$ are Klein forms of level $N([3],[4],[5],[6]$ and [11]). In a neighborhood of the cusp $i \infty$ of $\Gamma\left(2 N^{2}\right)$, the function $X_{r}(\tau)$ has an infinite product expansion:

$$
X_{r}\left(\frac{\tau}{2}\right):=q^{\frac{(r-1)(r+1-N)}{2 N}} \frac{1-q^{r}}{1-q} \prod_{m=1}^{\infty} \frac{\left(1-q^{N m-r}\right)\left(1-q^{N m+r}\right)}{\left(1-q^{N m-1}\right)\left(1-q^{N m+1}\right)} .
$$

Replacing $q$ by $q^{\frac{1}{N}}$, we obtain the usual form

$$
X_{\frac{r}{N}}\left(\frac{\tau}{2 N}\right):=q^{\frac{(r-1)(r+1-N)}{2 N^{2}}} \frac{1-q^{\frac{r}{N}}}{1-q^{\frac{1}{N}}} \prod_{m=1}^{\infty} \frac{\left(1-q^{n-\frac{r}{N}}\right)\left(1-q^{m+\frac{r}{N}}\right)}{\left(1-q^{m-\frac{1}{N}}\right)\left(1-q^{m+\frac{1}{N}}\right)} .
$$

Ishida proved that the coefficients of $X_{r}(\tau)$ at infinity are rational numbers $([4])$.

Let $\Gamma(N)$ denote the principal congruence subgroup of level $N$ of $S L_{2}(\mathbb{Z})$, in other words

$$
\Gamma(N)=\left\{\left(\begin{array}{ll}
a & b \\
c & d
\end{array}\right) \in S L_{2}(\mathbb{Z}) \mid a \equiv d \equiv 1, b \equiv c \equiv 0 \quad \bmod N\right\} .
$$

Let $F_{N}$ be the field of modular functions of level $N$ with rational Fourier coefficients. Denote $\bar{\Gamma}$ be the set $\Gamma /\left\{ \pm\left(\begin{array}{ll}1 & 0 \\ 0 & 1\end{array}\right)\right\}$ (respectively, $\Gamma$ ) if $\left(\begin{array}{cc}-1 & 0 \\ 0 & -1\end{array}\right) \in \Gamma$ (respectively, if $\left(\begin{array}{cc}-1 & 0 \\ 0 & -1\end{array}\right)$ is not in $\Gamma$ for a congruence subgroup $\Gamma$. Then $\left[F_{N}: F_{1}\right]=[\Gamma(1): \overline{\Gamma(N)}]<\infty$. Since $F_{1}=\mathbb{Q}(j), f$ is algebraic over $\mathbb{Q}(j)$ with $j$-invariant for each $f \in F_{N}$. Thus if $\alpha \in \mathfrak{H} \cap k$, then $f(\alpha)$ is algebraic over $\mathbb{Q}(j(\alpha))$ so that $f(\alpha)$ is algebraic over $\mathbb{Q}$. Thus we get the following:

Lemma 3.1. Let $\tau \in k \cap \mathfrak{H}$. Then the values of

$$
X_{r}\left(\frac{\tau}{2}\right):=q^{\frac{(r-1)(r+1-N)}{2 N}} \frac{1-q^{r}}{1-q} \prod_{m=1}^{\infty} \frac{\left(1-q^{N m-r}\right)\left(1-q^{N m+r}\right)}{\left(1-q^{N m-1}\right)\left(1-q^{N m+1}\right)}
$$

are algebraic numbers with $N \geq 6$.

Theorem 3.2. Let $\tau \in k \cap \mathfrak{H}$ and $n, t$ positive integers with $n>1$. Then the values of $q^{\frac{n}{12}-\frac{t}{2}+\frac{t^{2}}{2 n}} \prod_{m=1}^{\infty}\left(1 \pm q^{n m-t}\right)\left(1 \pm q^{n m-(n-t)}\right)$ are algebraic numbers with $1 \leq t<n$ and double signs in same order.

In the above product, the exponent of $q$ can be written as second Bernoulli polynomial $B_{2}(x)=x^{2}-x+\frac{1}{6}$, i.e., $\frac{n}{12}-\frac{t}{2}+\frac{t^{2}}{2 n}=\frac{n}{2} B_{2}\left(\frac{t}{n}\right)$. 
Proof. Note that $q^{\frac{n}{12}-\frac{t}{2}+\frac{t^{2}}{2 n}} \prod_{m=1}^{\infty}\left(1-q^{n m-t}\right)\left(1-q^{n m-(n-t)}\right)$ is algebraic number when $n=2,3,4,5([10])$. Hence we may consider the cases $n \geq 6$.

It is easy to see that

$$
\prod_{t=1}^{\frac{n-1}{2}} q^{\frac{n}{12}-\frac{t}{2}+\frac{t^{2}}{2 n}} \prod_{m=1}^{\infty}\left(1-q^{n m-t}\right)\left(1-q^{n m-(n-t)}\right)=\prod_{m=1}^{\infty} \frac{q^{\frac{1}{24}}\left(1-q^{m}\right)}{q^{\frac{n}{24}}\left(1-q^{n m}\right)}
$$

is an algebraic number by Proposition 1.1 and 1.2. Similarly we get that

$$
\begin{aligned}
& \prod_{t=1}^{\frac{n-2}{2}} q^{\frac{n}{12}-\frac{t}{2}+\frac{t^{2}}{2 n}} \prod_{m=1}^{\infty}\left(1-q^{n m-t}\right)\left(1-q^{n m-(n-t)}\right) \\
= & \prod_{m=1}^{\infty}\left(\frac{q^{\frac{1}{24}}\left(1-q^{m}\right)}{q^{\frac{n}{24}}\left(1-q^{n m}\right)}\right)\left(\frac{q^{\frac{n}{24}}}{\left(1-q^{n m-\frac{n}{2}}\right)}\right)
\end{aligned}
$$

is also an algebraic number for an even integer $n$. By Lemma 2.1, we know that

$$
\begin{aligned}
& q^{\frac{n}{12}-\frac{t}{2}+\frac{t^{2}}{2 n}} \prod_{m=1}^{\infty}\left(1-q^{n m-t}\right)\left(1-q^{n m-(n-t)}\right) \\
= & \alpha_{t} q^{\frac{n}{12}-\frac{1}{2}+\frac{1}{2 n}} \prod_{m=1}^{\infty}\left(1-q^{n m-1}\right)\left(1-q^{n m-(n-1)}\right)
\end{aligned}
$$

where $\alpha_{t}$ is an algebraic number and $t>2$. From $(3.1) \sim(3.3)$ and Proposition 2.3 , we get the theorem.

We shall also have occasion to use the Gauss polynomials:

$$
\left[\begin{array}{c}
m \\
n
\end{array}\right]= \begin{cases}\frac{(q)_{m}}{(q)_{n}(q)_{m-n}} & (0 \leq n \leq m) \\
0 & \text { otherwise. }\end{cases}
$$

We define

$$
\begin{gathered}
f_{n}(u)=\sum_{k=0}^{n}\left[\begin{array}{l}
n \\
k
\end{array}\right] u^{k}, \\
Q_{n}\left(u_{1}, u_{2}, \cdots, u_{k}\right)=\sum_{r_{1}+\cdots+r_{k}=n} \frac{(q)_{n}}{(q)_{r_{1}} \cdots(q)_{r_{k}}} u_{1}^{r_{1}} \cdots u_{k}^{r_{k}}
\end{gathered}
$$

and

$$
W(a, u):=\sum_{n=0}^{\infty} \frac{f_{n}(u)}{(q)_{n}}(a q)^{n}=\frac{1}{(a q)_{\infty}(a u q)_{\infty}} .
$$

By Theorem 3.2, we get the following:

$$
q^{-\left(\frac{1}{12}-\frac{2 k}{n}+\frac{k^{2}}{2 n^{2}}\right)} W\left(q^{-\frac{k}{n}}, q^{\frac{2 k}{n}-1}\right)=q^{-\left(\frac{1}{12}-\frac{2 k}{n}+\frac{k^{2}}{2 n^{2}}\right)} \sum_{m=0}^{\infty} \frac{f_{m}\left(q^{\frac{2 k}{n}-1}\right)}{(q)_{m}} q^{m-\frac{m k}{n}}
$$


and

$$
q^{-\sum_{i=1}^{s}\left(\frac{1}{12}-\frac{2 k_{i}}{n}+\frac{k_{i}^{2}}{2 n^{2}}\right)} \sum_{m=0}^{\infty} \frac{Q_{m}\left(q^{\frac{k_{1}}{n}}, q^{1-\frac{k_{1}}{n}}, \cdots, q^{\frac{k_{s}}{n}}, q^{1-\frac{k_{s}}{n}}\right)}{(q)_{m}}
$$

are algebraic numbers, where $1 \leq k, k_{i}<n$ and $n, k, k_{i} \in \mathbb{Z}$.

For a lattice $L$ in $\mathbb{C}$, the Weierstrass $\wp$-function is defined by

$$
\wp(\tau ; L)=\frac{1}{\tau^{2}}+\sum_{\omega \in L-\{0\}}\left\{\frac{1}{(\tau-\omega)^{2}}-\frac{1}{\omega^{2}}\right\}
$$

for $\tau \in \mathbb{C}$. Furthermore the Weierstrass $\wp$-function is defined by

$$
\sigma(\tau ; L)=\tau \prod_{\omega \in L-\{0\}}\left(1-\frac{\tau}{\omega}\right) e^{\frac{\tau}{\omega}+\frac{1}{2}\left(\frac{\tau}{\omega}\right)^{2}}
$$

for $\tau \in \mathbb{C}$ and it is an odd function. Taking the logarithmic derivative yields the Weierstrass $\zeta$-function

$$
\zeta(\tau ; L)=\frac{\sigma^{\prime}(\tau ; L)}{\sigma(\tau ; L)}=\frac{1}{\tau}+\sum_{\omega \in L-\{0\}}\left(\frac{1}{\tau-\omega}+\frac{1}{\omega}+\frac{\tau}{\omega^{2}}\right)
$$

for $\tau \in \mathbb{C}$.

Differentiating the function $\zeta(\tau+\omega ; L)-\zeta(\tau ; L)$ for any $\omega \in L$ yields 0 because $\zeta^{\prime}(\tau ; L)=\wp(\tau ; L)$ and the $\wp$-function is periodic. Hence there is a constant $\eta(\omega ; L)$ such that $\zeta(\tau+\omega ; L)=\zeta(\tau ; L)+\eta(\omega ; L)$. For $\left(r_{1}, r_{2}\right) \in \mathbb{Q}^{2}-\mathbb{Z}^{2}$, we can choose integers $u, v, N$ such that $\left(r_{1}, r_{2}\right)=\left(\frac{u}{N}, \frac{v}{N}\right)$. So, we define a Klein form $K_{r, s}$ of level $N$ by

$$
K_{u, v}(\tau)=e^{-\frac{1}{2 N^{2}}\left(u \eta_{1}+v \eta_{2}\right)(u \tau+v)} \sigma_{u, v}(\tau)
$$

where $\tau \in \mathbb{C}, \eta_{1}=\eta(\tau ;[\tau, 1]), \eta_{2}=\eta(1 ;[\tau, 1])$ and $\sigma_{u, v}=\sigma\left(\frac{u}{N} \tau+\frac{v}{N} ;[\tau, 1]\right)$. Note that $\eta_{1}$ and $\eta_{2}$ satisfy Legendre relation $\eta_{2} \tau-\eta_{1}=2 \pi i$.

As in [11], we use $\mathfrak{k}_{\frac{u}{N}, \frac{v}{N}}$ for the Klein form $K_{u, v}$ of level $N$, the Siegel function is defined by

$$
g_{\left(\frac{u}{N}, \frac{v}{N}\right)}(\tau)=\mathfrak{k}_{\left(\frac{u}{N}, \frac{n}{N}\right)}(\tau) \eta^{2}(\tau),
$$

where $\tau \in \mathbb{C}$ and $\eta(\tau)$ is a complex valued function defined as $\eta(\tau)=\sqrt{2 \pi i} e^{\frac{2 \pi i \tau}{24}}$ $\prod_{n=1}^{\infty}\left(1-e^{2 \pi i \tau n}\right)^{2}$. And $\eta^{24}(\tau)$ is a modular form of weight 12 for $\Gamma(1)$.

Remark 3.3. In this notation, we can rewrite the function defined by Ishida as $X_{r}\left(\frac{\tau}{2}\right)=\frac{g_{\left(\frac{r}{N}, 0\right)}}{g_{\left(\frac{1}{N}, 0\right)}}\left(\frac{N \tau}{2}\right)=\frac{\mathfrak{k}_{\left(\frac{r}{N}, 0\right)}}{\mathfrak{k}_{\left(\frac{1}{N}, 0\right)}}\left(\frac{N \tau}{2}\right)$. In this sense, the Siegel function can be written as

$$
g_{\left(\frac{t}{n}, 0\right)}\left(\frac{n \tau}{2}\right)=-q^{\frac{n}{2}-\frac{t}{2}+\frac{t^{2}}{2}} \prod_{m=1}^{\infty}\left(1-q^{n m-t}\right)\left(1-q^{n m-(n-t)}\right),
$$

where $q=e^{\pi i \tau}$ and it is a function in Theorem 2.2. Let $\mathfrak{H}$ be the complex upper half plane and $d$ be a positive square free integer. It is well-known fact 
that the value $g_{\left(r_{1}, r_{2}\right)}(\tau)$ for the Siegel function $g_{\left(r_{1}, r_{2}\right)}$ and $\tau \in \mathfrak{H} \cap \mathbb{Q}(\sqrt{-d})$ is an algebraic number. Using this, we can prove Theorem 3.2 as a modular function method.

\section{Proofs for our main theorems}

In this section, we prove our main theorems introduced in $\S 1$. To expand the definition $(a ; q)_{n}$ for a negative integer $n$, we redefine it as $(a)_{n}=(a q)_{n}=$ $\frac{(a ; q)_{\infty}}{\left(a q^{n} ; q\right)_{\infty}}=\frac{(a)_{\infty}}{\left(a q^{n}\right)_{\infty}}$ for $n \in \mathbb{Z}$.

Proof of Theorem 1.1. Let $l$ and $t$ be rational numbers and $0<l-t<1$. And $q=\exp (\pi i \tau)$. In (1.2), consider the case $z=q$,

$$
\begin{aligned}
\sum_{n=-\infty}^{\infty} \frac{(a)_{n}}{(b)_{n}} q^{n} & =\frac{(a q)_{\infty}\left(a^{-1}\right)_{\infty}\left(b a^{-1}\right)_{\infty}}{\left(b a^{-1} q^{-1}\right)_{\infty}(b)_{\infty}\left(a^{-1} q\right)_{\infty}} \\
& =\frac{\left(1-a^{-1}\right)}{\left(1-b a^{-1} q^{-1}\right)} \frac{\left(1-a^{-1}\right)(a q)_{\infty}}{\left(1-b a^{-1} q^{-1}\right)(b)_{\infty}} \\
& =-\frac{1}{a\left(1-b a^{-1} q^{-1}\right)} \frac{(a)_{\infty}}{(b)_{\infty}} .
\end{aligned}
$$

Then $\left|q^{1+l-t}\right|<|q|<1$. Applying $a=q^{t}$ and $b=q^{l+1}$ and $z=q$ in (4.1),

$$
\sum_{n=-\infty}^{\infty} \frac{\left(q^{t}\right)_{n}}{\left(q^{l+1}\right)_{n}} q^{n}=-\frac{\left(1-q^{l}\right)}{q^{t}\left(1-q^{l-t}\right)} \frac{\left(q^{t}\right)_{\infty}}{\left(q^{l}\right)_{\infty}} .
$$

Hence, we get

$$
\frac{\left(q^{t}\right)_{\infty}}{\left(q^{l}\right)_{\infty}}=-\frac{q^{t}\left(1-q^{l-t}\right)}{\left(1-q^{l}\right)} \sum_{n=-\infty}^{\infty} \frac{\left(q^{t}\right)_{n}}{\left(q^{l+1}\right)_{n}} q^{n}
$$

Similarly considering $a=q^{1-l}, b=q^{2-t}$ and $z=q$ in (4.1),

$$
\sum_{n=-\infty}^{\infty} \frac{\left(q^{1-l}\right)_{n}}{\left(q^{2-t}\right)_{n}} q^{n}=-\frac{\left(1-q^{1-t}\right)}{q^{1-l}\left(1-q^{l-t}\right)} \frac{\left(q^{1-l}\right)_{\infty}}{\left(q^{1-t}\right)_{\infty}}
$$

and

$$
\frac{\left(q^{1-l}\right)_{\infty}}{\left(q^{1-t}\right)_{\infty}}=-\frac{q^{1-l}\left(1-q^{l-t}\right)}{\left(1-q^{1-t}\right)} \sum_{n=-\infty}^{\infty} \frac{\left(q^{1-l}\right)_{n}}{\left(q^{2-t}\right)_{n}} q^{n} .
$$

Furthermore, since $\left|q^{2-l+t}\right|<|q|<1$, letting $a=q^{-t}$ and $b=q^{2-l}$ in (4.1),

$$
\sum_{n=-\infty}^{\infty} \frac{\left(q^{-t}\right)_{n}}{\left(q^{2-l}\right)_{n}} q^{n}=\frac{\left(1-q^{t}\right)\left(1-q^{1-l}\right)}{\left(1-q^{1-l+t}\right)} \frac{\left(q^{1-t}\right)_{\infty}}{\left(q^{1-l}\right)_{\infty}} .
$$


So,

$$
\frac{\left(q^{1-t}\right)_{\infty}}{\left(q^{1-l}\right)_{\infty}}=\frac{\left(1-q^{1-l+t}\right)}{\left(1-q^{t}\right)\left(1-q^{1-l}\right)} \sum_{n=-\infty}^{\infty} \frac{\left(q^{-t}\right)_{n}}{\left(q^{2-l}\right)_{n}} q^{n}
$$

On the other hand, since $|q|<1$ by (1.1) and (1.1.1),

$$
\begin{aligned}
& f\left(-q^{t},-q^{1-t}\right)=\sum_{n=-\infty}^{\infty}(-1)^{n} q^{\frac{n}{2}(n-1+2 t)}=\left(q^{t}\right)_{\infty}\left(q^{1-t}\right)_{\infty}(q)_{\infty}, \\
& f\left(-q^{l},-q^{1-l}\right)=\sum_{n=-\infty}^{\infty}(-1)^{n} q^{\frac{n}{2}(n-1+2 l)}=\left(q^{l}\right)_{\infty}\left(q^{1-l}\right)_{\infty}(q)_{\infty} .
\end{aligned}
$$

Then using (4.2) and (4.4),

$$
\begin{gathered}
\prod_{n=1}^{\infty} \frac{\left(1-q^{n-t}\right)\left(1-q^{n-1+t}\right)}{\left(1-q^{n-l}\right)\left(1-q^{n-1+l}\right)}=\frac{\left(q^{t}\right)_{\infty}}{\left(q^{l}\right)_{\infty}} \cdot \frac{\left(q^{1-t}\right)_{\infty}}{\left(q^{1-l}\right)_{\infty}} \\
=-\frac{q^{t}\left(1-q^{l-t}\right)\left(1-q^{1-l+t}\right)}{\left(1-q^{t}\right)\left(1-q^{l}\right)\left(1-q^{1-l}\right)} \sum_{n, m=-\infty}^{\infty} \frac{\left(q^{t}\right)_{n}\left(q^{-t}\right)_{m}}{\left(q^{1+l}\right)_{n}\left(q^{2-l}\right)_{m}} q^{n+m} .
\end{gathered}
$$

Now by (4.2) and (4.3)

$$
\frac{\left(q^{t}\right)_{\infty}}{\left(q^{l}\right)_{\infty}} \cdot \frac{\left(q^{1-t}\right)_{\infty}}{\left(q^{1-l}\right)_{\infty}}=q^{l+t-1} \frac{\left(1-q^{1-t}\right)}{\left(1-q^{l}\right)} \frac{\sum_{n=-\infty}^{\infty} \frac{\left(q^{t}\right)_{n}}{\left(q^{1+l}\right)_{n}} q^{n}}{\sum_{n=-\infty}^{\infty} \frac{\left(q^{1-l}\right)_{n}}{\left(q^{2-t}\right)_{n}} q^{n}}
$$

and from (4.5) and (4.6)

$$
\begin{aligned}
\frac{\left(q^{t}\right)_{\infty}\left(q^{1-t}\right)_{\infty}(q)_{\infty}}{\left(q^{1-l}\right)_{\infty}\left(q^{l}\right)_{\infty}(q)_{\infty}} & =\frac{f\left(-q^{t},-q^{1-t}\right)}{f\left(-q^{l},-q^{1-l}\right)} \\
& =\frac{\sum_{n=-\infty}^{\infty}(-1)^{n} q^{\frac{1}{2} n(n-1+2 t)}}{\sum_{n=-\infty}^{\infty}(-1)^{n} q^{\frac{1}{2} n(n-1+2 l)}} .
\end{aligned}
$$

Through the above, we get three identities. It is sufficient to show that the result multiplying the equations of $(4.7) \sim(4.9)$ to $q^{\frac{t(t-1)-l(l-1)}{2}}$ is algebraic number. Using the simplest form it is just

$$
\begin{aligned}
& q^{\frac{t(t-1)-l(l-1)}{2}} \prod_{n=1}^{\infty} \frac{\left(1-q^{n-t}\right)\left(1-q^{n-1+t}\right)}{\left(1-q^{n-l}\right)\left(1-q^{n-1+l}\right)} \\
= & \frac{q^{\frac{1}{12}-\frac{t}{2}+\frac{t^{2}}{2}}}{q^{\frac{1}{12}-\frac{l}{2}+\frac{l^{2}}{2}}} \prod_{n=1}^{\infty} \frac{\left(1-q^{n}-t\right)\left(1-q^{n}-1+t\right)}{\left(1-q^{n-l}\right)\left(1-q^{n-1+l}\right)}
\end{aligned}
$$

and by Theorem 3.2 both denominator and numerator are algebraic numbers for $\tau \in k \cap \mathfrak{H}$.

Remark 4.1. Theorem 1.1 gives the following identities between two infinite series:

$$
q^{l+t-1} \frac{1-q^{1-t}}{1-q^{l}} \sum_{n=-\infty}^{\infty} \frac{\left(q^{t}\right)_{n}}{\left(q^{1+l}\right)_{n}} q^{n}=\frac{\left(q^{t}\right)_{\infty}\left(q^{1-t}\right)_{\infty}}{\left(q^{l}\right)_{\infty}\left(q^{1-l}\right)_{\infty}} \sum_{n=-\infty}^{\infty} \frac{\left(q^{1-l}\right)_{n}}{\left(q^{2-t}\right)_{n}} q^{n}
$$


Proof of Theorem 1.2. Consider (1.2) when $b=q$ and $\left|\frac{q}{a}\right|<|z|<1$

$$
\sum_{n=-\infty}^{\infty} \frac{(a)_{n}}{(q)_{n}} z^{n}=\sum_{n=0}^{\infty} \frac{(a)_{n}}{(q)_{n}} z^{n}=\frac{(a z)_{\infty}}{(z)_{\infty}}
$$

because $\frac{1}{(q)_{n}}=\frac{\left(q^{n+1}\right)_{\infty}}{(q)_{\infty}}=\frac{\left(1-q^{n+1}\right) \cdots\left(1-q^{n+(-n)}\right) \cdots}{(q)_{\infty}}=0$ for a negative integer $n$. Since $l$ and $t$ are the rational numbers with $0<t, l, t+l<1$, we can check the convergence condition and obtain (4.12) (respectively, (4.13) and (4.14)) when $a=q^{t}$ (respectively, $a=q^{-t}$ and $t$ ) and $z=q^{l}$ (respectively, $z=q^{1-l}$ and $\left.z=q^{1-t-m}\right)$ in $(4.11)$ :

$$
\begin{gathered}
\sum_{n=0}^{\infty} \frac{\left(q^{t}\right)_{n}}{(q)_{n}} q^{l n}=\frac{\left(q^{t+l}\right)_{\infty}}{\left(q^{l}\right)_{\infty}} \\
\sum_{n=0}^{\infty} \frac{\left(q^{-t}\right)_{n}}{(q)_{n}} q^{(1-l) n}=\frac{\left(q^{1-t-l}\right)_{\infty}}{\left(q^{1-l}\right)_{\infty}} \\
\sum_{n=0}^{\infty} \frac{\left(q^{t}\right)_{n}}{(q)_{n}} q^{(1-t-l) n}=\frac{\left(q^{1-l}\right)_{\infty}}{\left(q^{1-l-t}\right)_{\infty}}
\end{gathered}
$$

Multiplying (4.12) by (4.13) and dividing (4.12) by (4.14), we obtain that

$$
\text { (4.15) } \begin{aligned}
\prod_{n=1}^{\infty} \frac{\left(1-q^{n-t-l}\right)\left(1-q^{n-1+t+l}\right)}{\left(1-q^{n-l}\right)\left(1-q^{n-1+l}\right)} & =\frac{\left(q^{t+l}\right)_{\infty}\left(q^{1-t-l}\right)_{\infty}}{\left(q^{l}\right)_{\infty}\left(q^{1-l}\right)_{\infty}} \\
& =\sum_{m, n=0}^{\infty} \frac{\left(q^{t}\right)_{n}\left(q^{-t}\right)_{m}}{(q)_{n}(q)_{m}} q^{l(n-m)+m} \\
& =\frac{\sum_{n=0}^{\infty} \frac{\left(q^{t}\right)_{n}}{(q)_{n}} q^{l n}}{\sum_{n=0}^{\infty} \frac{\left(q^{t}\right)_{n}}{(q)_{n}} q^{(1-t-l) n}} \\
& =\frac{f\left(-q^{t+l},-q^{1-t-l}\right)}{f\left(-q^{l},-q^{1-l}\right)} \\
& =\frac{\sum_{n=-\infty}^{\infty}(-1)^{n} q^{\frac{n}{2}(n-1+2 t+2 l)}}{\sum_{n=-\infty}^{\infty}(-1)^{n} q^{\frac{n}{2}(n-1+2 l)}}
\end{aligned}
$$


where last equality is given by (1.2). Multiplying the factor $q^{\frac{t}{2}(2 l+t-1)}$ and using Theorem 3.2 make our assertion true:

$$
\begin{aligned}
& \frac{q^{\frac{1}{12}-\frac{t+l}{2}+\frac{(t+l)^{2}}{2}}}{q^{\frac{1}{12}-\frac{l}{2}+\frac{l^{2}}{2}}} \prod_{n=1}^{\infty} \frac{\left(1-q^{n-t-l}\right)\left(1-q^{n-1+t+l}\right)}{\left(1-q^{n-l}\right)\left(1-q^{n-1+l}\right)} \\
= & q^{\frac{t}{2}(2 l+t-1)} \sum_{n, m=0}^{\infty} \frac{\left(q^{t}\right)_{n}\left(q^{-t}\right)_{m}}{(q)_{n}(q)_{m}} q^{l(n-m)+m} \\
= & q^{\frac{t}{2}(2 l+t-1)} \frac{\sum_{n=0}^{\infty} \frac{\left(q^{t}\right)_{n}}{(q)_{n}} q^{l n}}{\sum_{n=0}^{\infty} \frac{\left(q^{t}\right)_{n}}{(q)_{n}} q^{(1-t-l) n}}
\end{aligned}
$$

Proof of Theorem 1.3. Now we put $b=a q z$ in (1.2):

$$
\begin{aligned}
\sum_{n=-\infty}^{\infty} \frac{(a)_{n}}{(a q z)_{n}} z^{n} & =\frac{(a z)_{\infty}\left(a^{-1} q z^{-1}\right)_{\infty}(q z)_{\infty}}{(z)_{\infty}(a q z)_{\infty}\left(a^{-1} q\right)_{\infty}} \\
& =\frac{1-a z}{1-z} \frac{\left(a^{-1} q z^{-1}\right)_{\infty}}{\left(a^{-1} q\right)_{\infty}}
\end{aligned}
$$

Since $0<l<1$, applying $a=q^{t}, z=q^{l}$ (respectively, $a=q^{-t}, z^{1-l}$ and $a=q^{1-t-l}, z=q^{l}$ ) gives us (4.17) (respectively, (4.18) and (4.19)).

$$
\begin{aligned}
& \sum_{n=-\infty}^{\infty} \frac{\left(q^{t}\right)_{n}}{\left(q^{1+t+l}\right)_{n}} q^{l n}=\frac{\left(1-q^{m+t}\right)}{\left(1-q^{m}\right)} \frac{\left(q^{1-t-m}\right)_{\infty}}{\left(q^{1-t}\right)_{\infty}}, \\
& \sum_{n=-\infty}^{\infty} \frac{\left(q^{-t}\right)_{n}}{\left(q^{2-t-l}\right)_{n}} q^{(1-l) n}=\frac{\left(1-q^{t}\right)\left(1-q^{1-l-t}\right)}{\left(1-q^{1-l}\right)} \frac{\left(q^{t+l}\right)_{\infty}}{\left(q^{t}\right)_{\infty}} \\
& \sum_{n=-\infty}^{\infty} \frac{\left(q^{1-t-l}\right)_{n}}{\left(q^{2-t}\right)_{n}} q^{l n}=\frac{\left(1-q^{1-t}\right)}{\left(1-q^{l}\right)} \frac{\left(q^{t}\right)_{\infty}}{\left(q^{t+l}\right)_{\infty}} .
\end{aligned}
$$


In a similar way, we derive that

$$
\text { 20) } \begin{aligned}
& \prod_{n=1}^{\infty} \frac{\left(1-q^{n-t-l}\right)\left(1-q^{n-1+t+l}\right)}{\left(1-q^{n-t}\right)\left(1-q^{n-1+t}\right)}=\frac{\left(q^{1-t-l}\right)_{\infty}\left(q^{t+l}\right)_{\infty}}{\left(q^{1-t}\right)_{\infty}\left(q^{t}\right)_{\infty}} \\
= & \frac{\left(1-q^{l}\right)\left(1-q^{1-l}\right)}{\left(1-q^{t}\right)\left(1-q^{1-l-t}\right)\left(1-q^{l+t}\right)} \sum_{n, m=-\infty}^{\infty} \frac{\left(q^{t}\right)_{n}\left(q^{-t}\right)_{m}}{\left(q^{1+t+l}\right)_{n}\left(q^{2-t-l}\right)_{m}} q^{l(n-m)+m} \\
= & \frac{\left(1-q^{1-t}\right)}{\left(1-q^{l+t}\right)} \frac{\sum_{n=-\infty}^{\infty} \frac{\left(q^{t}\right)_{n}}{\left.\sum_{n=-\infty}^{\infty+t+l}\right)_{n}} q^{l n}}{\left(q^{2-t}\right)_{n}} q^{l n} \\
= & \frac{f\left(-q^{t+l},-q^{1-t-l}\right)}{f\left(-q^{t},-q^{1-t}\right)} \\
= & \frac{\sum_{n=-\infty}^{\infty}(-1)^{n} q^{\frac{n}{2}(n-1+2 t+2 l)}}{\sum_{n=-\infty}^{\infty}(-1)^{n} q^{\frac{n}{2}(n-1+2 t)}} .
\end{aligned}
$$

After multiplying $q^{\frac{1}{2} l(l+2 t-1)}$ and by using Theorem 3.2 again, we get the quotient form of two algebraic numbers. Hence we are done.

Remark 4.2. (4.20) is different from (4.7) and (4.15). This is another formula for theta series.

Now consider the Rogers-Ramanujan continued fraction:

$$
R(q)=\frac{q^{\frac{1}{5}}}{1+\frac{q}{1+\frac{q^{2}}{1+\cdots}}}, \quad|q|<1
$$

First, let

$$
G(q):=\sum_{n=0}^{\infty} \frac{q^{n^{2}}}{(q ; q)_{n}} \quad \text { and } \quad H(q):=\sum_{n=0}^{\infty} \frac{q^{n(n+1)}}{(q ; q)_{n}} .
$$

Then it is the well-known fact that these satisfy the Rogers-Ramanujan identities $([13],[14])$ :

$$
G(q)=\frac{1}{\left(q ; q^{5}\right)_{\infty}\left(q^{4} ; q^{5}\right)_{\infty}} \quad \text { and } \quad H(q)=\frac{1}{\left(q^{2} ; q^{5}\right)_{\infty}\left(q^{3} ; q^{5}\right)_{\infty}} .
$$

Then Rogers proved that

in 1894 ([14]).

$$
R(q)=q^{\frac{1}{5}} \frac{H(q)}{G(q)}=q^{\frac{1}{5}} \frac{\left(q ; q^{5}\right)_{\infty}\left(q^{4} ; q^{5}\right)_{\infty}}{\left(q^{2} ; q^{5}\right)_{\infty}\left(q^{3} ; q^{5}\right)_{\infty}}
$$

From the identities in this section, we obtain the following identities for $R\left(q^{\frac{1}{5}}\right)$

$$
R\left(q^{\frac{1}{5}}\right)=q^{\frac{1}{25}} \frac{H\left(q^{\frac{1}{5}}\right)}{G\left(q^{\frac{1}{5}}\right)}=q^{\frac{1}{25}} \frac{f\left(-q^{\frac{1}{5}} ;-q^{\frac{4}{5}}\right)}{f\left(-q^{\frac{2}{5}} ;-q^{\frac{3}{5}}\right)}
$$


$=q^{\frac{1}{25}} \frac{\sum_{n=0}^{\infty} \frac{q^{\frac{1}{5} n(n+1)}}{\left(q^{\frac{1}{5}} ; q^{\frac{1}{5}}\right)_{n}}}{\sum_{n=0}^{\infty} \frac{q^{\frac{1}{5} n^{2}}}{\left(q^{\frac{1}{5}} ; q^{\frac{1}{5}}\right)_{n}}}$

$=-q^{\frac{6}{25}} \frac{\left(1+q^{\frac{2}{5}}\right)}{\left(1-q^{\frac{3}{5}}\right)} \sum_{n, m=-\infty}^{\infty} \frac{\left(q^{\frac{1}{5}} ; q\right)_{n}\left(q^{-\frac{1}{5}} ; q\right)_{m}}{\left(q^{\frac{7}{5}} ; q\right)_{n}\left(q^{\frac{8}{5}} ; q\right)_{m}} q^{n+m}$

(by $(4.7) ; t=\frac{1}{5}, l=\frac{2}{5}$ )

$=q^{-\frac{9}{25}}\left(1+q^{\frac{2}{5}}\right) \frac{\sum_{n=-\infty}^{\infty} \frac{\left(\frac{1}{5} ; q\right)_{n}}{\left(q^{\frac{7}{5}} ; q\right)_{n}} q^{n}}{\sum_{n=-\infty}^{\infty} \frac{\left(q^{\frac{3}{5}} ; q\right)_{n}}{\left(q^{\frac{9}{5}} ; q\right)_{n}} q^{n}} \quad$ (by $\left.(4.7) ; t=\frac{1}{5}, l=\frac{2}{5}\right)$

$=q^{\frac{1}{25}} \sum_{n, m=0}^{\infty} \frac{\left(q^{\frac{2}{5}} ; q\right)_{n}\left(q^{-\frac{2}{5}} ; q\right)_{m}}{(q ; q)_{n}(q ; q)_{m}} q^{\frac{2}{5}(n-m)+m} \quad\left(b y(4.15) ; t=l=\frac{2}{5}\right)$

$=q^{\frac{1}{25}} \sum_{n, j=0}^{\infty} \frac{\left(q^{\frac{3}{5}} ; q\right)_{n}\left(q^{-\frac{3}{5}} ; q\right)_{m}}{(q ; q)_{n}(q ; q)_{m}} q^{\frac{1}{5}(n-m)+m} \quad\left(\right.$ by $\left.(4.15) ; t=\frac{3}{5}, l=\frac{1}{5}\right)$

$=q^{\frac{1}{25}} \frac{\sum_{n=0}^{\infty} \frac{\left(q^{\frac{2}{5}} ; q\right)_{n}}{(q ; q)} q^{\frac{2}{5} n}}{\sum_{n=0}^{\infty} \frac{\left(q^{\frac{2}{5}} ; q\right)_{n}}{(q ; q)_{n}} q^{\frac{1}{5} n}} \quad$ (by $\left.(4.15) ; t=l=\frac{2}{5}\right)$

$=q^{\frac{1}{25}} \frac{\sum_{n=0}^{\infty} \frac{\left(q^{\frac{1}{5}} ; q\right)_{n}}{(q ; q)_{n}} q^{\frac{3}{5} n}}{\sum_{n=0}^{\infty} \frac{\left(q^{\frac{1}{5}} ; q\right)_{n}}{(q ; q)_{n}} q^{\frac{1}{5} n}} \quad \quad\left(\right.$ by $\left.(4.15) ; t=\frac{1}{5}, l=\frac{3}{5}\right)$

$=q^{\frac{1}{25}} \frac{\left(1+q^{\frac{1}{5}}+q^{\frac{2}{5}}\right)}{\left(1-q^{\frac{4}{5}}\right)} \sum_{n, m=-\infty}^{\infty} \frac{\left(q^{\frac{2}{5}} ; q\right)_{n}\left(q^{-\frac{2}{5}} ; q\right)_{m}}{\left(q^{\frac{9}{5}} ; q\right)_{n}\left(q^{\frac{6}{5}} ; q\right)_{m}} q^{\frac{2}{5} n+\frac{3}{5} m}$

(by $(4.20) ; t=l=\frac{2}{5}$ )

$=q^{\frac{1}{25}} \frac{1}{\left(1-q^{\frac{3}{5}}\right)} \sum_{n, j=-\infty}^{\infty} \frac{\left(q^{\frac{3}{5}} ; q\right)_{n}\left(q^{-\frac{3}{5}} ; q\right)_{m}}{\left(q^{\frac{9}{5}} ; q\right)_{n}\left(q^{\frac{6}{5}} ; q\right)_{m}} q^{\frac{1}{5} n+\frac{4}{5} m}$

(by $(4.20) ; t=\frac{3}{5}, l=\frac{1}{5}$ )

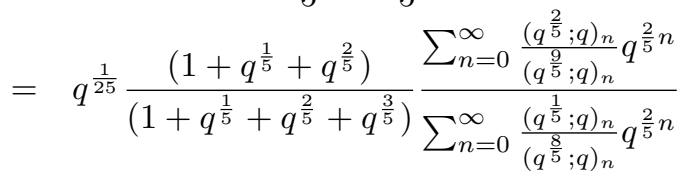

(by $(4.20) ; t=l=\frac{2}{5}$ ) 


$$
\begin{gathered}
=q^{\frac{1}{25}} \frac{1}{\left(1+q^{\frac{2}{5}}\right)} \frac{\sum_{n=0}^{\infty} \frac{\left(q^{\frac{3}{5}} ; q\right)_{n}}{\left(q^{\frac{9}{5}} ; q\right)_{n}} q^{\frac{1}{5} n}}{\sum_{n=0}^{\infty} \frac{\left(q^{\frac{1}{5}} ; q\right)_{n}}{\left(q^{\frac{7}{5}} ; q\right)_{n}} q^{\frac{1}{5} n}} . \\
\quad\left(\operatorname{by}(4.20) ; t=\frac{3}{5}, l=\frac{1}{5}\right)
\end{gathered}
$$

\section{Appell series}

Let

$$
\begin{aligned}
S \quad & \left(c ; b, b^{\prime} ; d ; a, a^{\prime} ; e, f ; x, y, x^{\prime}, y^{\prime}: q\right) \\
& :=\sum_{m, n, k, l=0}^{\infty} \frac{(c)_{m+n}(b)_{m}\left(b^{\prime}\right)_{n}(d)_{k+l}(a)_{k}\left(a^{\prime}\right)_{l}}{(q)_{m}(q)_{n}(q)_{k}(q)_{l}(e)_{m+n}(f)_{k+l}} x^{m} y^{n}\left(x^{\prime}\right)^{k}\left(y^{\prime}\right)^{l} \\
& =\Phi^{(1)}\left[c ; b, b^{\prime} ; e ; x, y: q\right] \Phi^{(1)}\left[d ; a, a^{\prime} ; f ; x^{\prime}, y^{\prime}: q\right] .
\end{aligned}
$$

From (1.3), we deduce that

$$
\Phi^{(1)}\left[\frac{b^{\prime}}{x} ; b, b^{\prime} ; b b^{\prime} ; x, y ; q\right]=\frac{(b x)_{\infty}\left(b^{\prime}\right)_{\infty}\left(b^{\prime} y / x\right)_{\infty}}{\left(b b^{\prime}\right)_{\infty}(x)_{\infty}(y)_{\infty}}
$$

and

$$
\Phi^{(1)}\left[\frac{d^{\prime}}{w} ; d, d^{\prime} ; d d^{\prime} ; w, s ; q\right]=\frac{(d w)_{\infty}\left(d^{\prime}\right)_{\infty}\left(d^{\prime} s / w\right)_{\infty}}{\left(d d^{\prime}\right)_{\infty}(w)_{\infty}(s)_{\infty}} .
$$

Let $b=q^{B}, b^{\prime}=q^{B^{\prime}}, d=q^{D}, d^{\prime}=q^{D^{\prime}}, x=q^{X}, y=q^{Y}, w=q^{W}$ and $s=q^{S}$, where $B^{\prime}+D^{\prime}=1, X+W=1, Y+S=1, B+D=1, B+X>1$ and $B+B^{\prime}>1$ with $0<X, Y, B, B^{\prime}, D, D^{\prime}, S, W<1$ rational numbers.

Thus, we get the following:

$$
\begin{aligned}
& S\left(q^{B^{\prime}-X} ; q^{B}, q^{B^{\prime}} ; q^{D^{\prime}-W} ; q^{D}, q^{D^{\prime}} ; q^{B+B^{\prime}}, q^{D+D^{\prime}}, q^{X}, q^{Y}, q^{W}, q^{S}: q\right) \\
= & \sum_{m, n, k, l=0}^{\infty} \frac{\left(q^{B^{\prime}-X}\right)_{m+n}\left(q^{B}\right)_{m}\left(q^{B^{\prime}}\right)_{n}\left(q^{D^{\prime}-W}\right)_{k+l}\left(q^{D}\right)_{k}\left(q^{D^{\prime}}\right)_{l}}{(q)_{m}(q)_{n}(q)_{k}(q)_{l}\left(q^{B+B^{\prime}}\right)_{m+n}\left(q^{D+D^{\prime}}\right)_{k+l}} q^{X+Y n+W k+S l} \\
= & \Phi^{(1)}\left[q^{B^{\prime}-X} ; q^{B}, q^{B^{\prime}} ; q^{B} q^{B^{\prime}} ; q^{X}, q^{Y} ; q\right] \Phi^{(1)}\left[q^{D^{\prime}-W} ; q^{D}, q^{D^{\prime}} ; q^{D} q^{D^{\prime}} ; q^{W}, q^{S} ; q\right] \\
= & \frac{\left(q^{B+X}\right)_{\infty}\left(q^{B^{\prime}}\right)_{\infty}\left(q^{B^{\prime}+Y-X}\right)_{\infty}\left(q^{D+W}\right)_{\infty}\left(q^{D^{\prime}}\right)_{\infty}\left(q^{D^{\prime}+S-W}\right)_{\infty}}{\left(q^{B+B^{\prime}}\right)_{\infty}\left(q^{X}\right)_{\infty}\left(q^{Y}\right)_{\infty}\left(q^{D+D^{\prime}}\right)_{\infty}\left(q^{W}\right)_{\infty}\left(q^{S}\right)_{\infty}} \\
= & \left(\frac{1-q^{B+B^{\prime}-1}}{1-q^{B+X-1}}\right) \\
& \cdot \frac{\left(q^{B+X-1}\right)_{\infty}\left(q^{B^{\prime}}\right)_{\infty}\left(q^{B^{\prime}+Y-X}\right)_{\infty}\left(q^{D+W}\right)_{\infty}\left(q^{D^{\prime}}\right)_{\infty}\left(q^{D^{\prime}+S-W}\right)_{\infty}}{\left(q^{B+B^{\prime}-1}\right)_{\infty}\left(q^{X}\right)_{\infty}\left(q^{Y}\right)_{\infty}\left(q^{D+D^{\prime}}\right)_{\infty}\left(q^{W}\right)_{\infty}\left(q^{S}\right)_{\infty}} \\
= & \left(\frac{1-q^{B+B^{\prime}-1}}{1-q^{B+X-1}}\right) \cdot \frac{f\left(-q^{B+X-1}\right) f\left(-q^{B^{\prime}}\right) f\left(-q^{B^{\prime}+Y-X}\right)}{f\left(-q^{B+B^{\prime}-1}\right) f\left(-q^{X}\right) f\left(-q^{Y}\right)}
\end{aligned}
$$




$$
\begin{aligned}
= & \left(\frac{1-q^{B+B^{\prime}-1}}{1-q^{B+X-1}}\right) \\
& \cdot \frac{W\left(q^{B+B^{\prime}-2}, q^{3-2\left(B+B^{\prime}\right)}\right) W\left(q^{X-1}, q^{1-2 X}\right) W\left(q^{Y-1}, q^{1-2 Y}\right)}{W\left(q^{B^{\prime}-1}, q^{1-2 B^{\prime}}\right) W\left(q^{B+X-2}, q^{3-2(B+X)}\right) W\left(q^{B^{\prime}+Y-X-1}, q^{1-2\left(B^{\prime}+Y-X\right)}\right)} .
\end{aligned}
$$

Since $B, B^{\prime}, X, Y \in \mathbb{Q}$, we find $N$ satisfying $N=\operatorname{lcm}\left(N_{1}, N_{2}, \cdots, N_{6}\right)$ with $B+X-1:=\frac{M_{1}}{N_{1}}, B^{\prime}:=\frac{M_{2}}{N_{2}}, B^{\prime}+Y-X:=\frac{M_{3}}{N_{3}}, B+B^{\prime}-1:=\frac{M_{4}}{N_{4}}, X:=\frac{M_{5}}{N_{5}}$, $Y=\frac{M_{6}}{N_{6}}$.

Here, $M_{i}, N_{j}(1 \leq i, j \leq 6) \in \mathbb{Z}$.

Using Theorem 3.2,

$$
\begin{aligned}
& q^{\left(B^{\prime}-X\right)\left(B^{\prime}-X+1\right)-B^{\prime}(B-Y)-X Y}\left(\frac{1-q^{B+X-1}}{1-q^{B+B^{\prime}-1}}\right) . \\
& S\left(q^{B^{\prime}-X} ; q^{B}, q^{B^{\prime}} ; q^{D^{\prime}-W} ; q^{D}, q^{D^{\prime}} ; q^{B+B^{\prime}}, q^{D+D^{\prime}}, q^{X}, q^{Y}, q^{W}, q^{S}: q\right) \\
& =\frac{X_{\frac{M_{1} N}{N_{1}} / N}\left(\frac{\tau}{2 N}\right) X_{\frac{M_{2} N}{N_{2}} / N}\left(\frac{\tau}{2 N}\right) X_{\frac{M_{3} N}{N_{3}} / N}\left(\frac{\tau}{2 N}\right)}{X_{\frac{M_{4} N}{N_{4}} / N}\left(\frac{\tau}{2 N}\right) X_{\frac{M_{5} N}{N_{5}} / N}\left(\frac{\tau}{2 N}\right) X_{\frac{M_{6} N}{N_{6}} / N}\left(\frac{\tau}{2 N}\right)}
\end{aligned}
$$

is an algebraic number.

Replace $q$ by $q^{N}$, we get that $S, \frac{f(t)}{f(s)}, \frac{W(a, b)}{W(c, d)}$ have infinite product expansions in a neighborhood of the cusp $i \infty$ of $\Gamma\left(2 N^{2}\right)$. So we can deduce that special cases of basic Appell series has an infinite product expansion in a neighborhood of the cusp $i \infty$ of $\Gamma\left(2 N^{2}\right)$.

\section{References}

1. G. E. Andrews, Summations and transformations for basic Appell series, J. London Math. Soc. (2) 4 (1972), 618-622.

2. B. C. Berndt, Ramanujan's notebooks. Part III, Springer-Verlag, 1991.

3. N. Ishida, Generators and equations for modular function fields of principal congruence subgroups, Acta Arith. 85 (1998), no. 3, 197-207.

4. N. Ishida and N. Ishii, Generators and defining equation of the modular function field of the group $\Gamma_{1}(N)$, Acta Arith. 101 (2002), no. 4, 303-320.

5. N. Ishida and N. Ishii, The equations for modular function fields of principal congruence subgroups of prime level, Manuscripta Math. 90 (1996), no. 3, 271-285.

6. N. Ishida and N. Ishii, The equation for the modular curve $X_{1}(N)$ derived from the equation for the modular curve $X(N)$, Tokyo J. Math. 22 (1999), no. 1, 167-175.

7. F. J. Jackson, On basic double hypergeometric functions, Quart. J. Math., 13 (1942), 69-82.

8. F. J. Jackson, q-identities of Auluck, Carlitz, and Rogers, Quart. J. Math., 15 (1944), 49-61.

9. D. Kim and J. K. Koo, Algebraic integers as values of elliptic functions, Acta Arith. 100 (2001), 105-116.

10. D. Kim and J. K. Koo, On the infinite products derived from theta series I, J. Korean Math. Soc. 44 (2007), no. 1, 55-107.

11. D. Kubert and S. Lang, Units in the modular function field, Math. Ann, 218 (1975), 175-189.

12. S. Lang, Elliptic Functions, Addison-Wesley, 1973. 
13. S. Ramanujan, Proof of certain identities in combinatory analysis, Proc. Cambridge Philos. Soc. 19 (1919), 214-216.

14. L. J. Rogers, Second memoir on the expansion of certain infinite products, Proc. London Math. Soc. 25(1984), 318-343.

GYEONG - SIG SEO

Department of Mathematics and

Institute of Pure and Applied Mathematics

Chonbuk National University

Chonju 561-756, Korea

E-mail: sgs0303@chonbuk.ac.kr

Joong-Soo Park

Department of Mathematics Education

Woosuk University

Wanju 565-701, Korea

E-mail: jspark@woosuk.ac.kr 